\title{
Contrôle central de la formation osseuse
}

\section{Gérard Karsenty}

\section{ADRESSE}

G. Karsenty : Department of Molecular and Human Genetics, Baylor College of Mediçine, One Baylor Place, Houston TX 77030, États-Unis.

L'observation que l'arrêt des fonctions gonadiques favorise l'apparition de l'ostéoporose alors que l'obésité prévient la perte osseuse nous a amenés à postuler que la masse osseuse, les fonctions gonadiques et la reproduction sont contrôlées par les mêmes hormones. Une analyse in vivo utilisant des méthodes génétiques et physiologiques a permis de démontrer que la leptine est le plus puissant inhibiteur de la formation osseuse identifié à ce jour. Les mêmes études in vivo ont permis de démontrer également que cette fonction de la leptine, comme les autres, requiert sa liaison à un récepteur hypothalamique. Cette étude démontre que, comme la plupart des autres fonctions homéostatiques, le remodelage osseux est sous le contrôle de l'hypothalamus. Cette étude suggère aussi que les maladies osseuses dégénératives telle que l'ostéoporose pourraient être en partie d'origine hypothalamique.

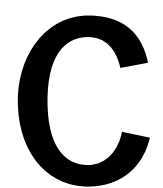
hez les vertébrés la masse osseuse reste constante à la fin de la période de croissance, grâce à un processus complexe et dynamique appelé remodelage osseux [1]. La première étape, rapide, se déroulant en quelques semaines, consiste en une résorption de l'os pré-existant par les ostéoclastes. Puis intervient une étape de formation osseuse de novo, plus lente, pouvant durer plusieurs mois, assurée par les ostéoblastes. Un cycle de remodelage osseux typique s'étale donc sur au moins trois mois. De tels cycles de destruction/formation se déroulent simultanément dans plusieurs sites osseux, de sorte que la masse osseuse totale d'un individu reste constante jusqu'aux stades tardifs de la vie. Ainsi, chez la femme, la masse osseuse reste constante jusqu'à la ménopause. C'est l'insuffisance de la production d'hormones sexuelles qui est à l'origine d'une augmentation relative de la résorption par rapport à la formation osseuse, déséquilibre qui conduit à une diminution de la masse osseuse (ostéopénie) et expose au risque potentiel de fractures provoquées par des traumatismes minimes (ostéoporose) [1]. L'importance biologique du remodelage osseux est illustrée par le fait que l'ostéoporose est la maladie dégénérative la plus fréquente des pays développés, touchant par exemple 28 millions de personnes aux États-Unis. De plus, le vieillissement progressif de la 
population explique que son incidence soit en constante augmentation.

\section{Le remodelage osseux: une fonction complexe mal comprise}

La simultanéité du remodelage osseux dans de nombreux sites a longtemps été utilisée comme un argument en faveur d'une régulation locale, autocrine ou paracrine, de cette fonction physiologique [2]. Cette hypothèse est corroborée par des preuves expérimentales. L'une d'elles, et non des moindres, est la démonstration que la différenciation des ostéoclastes, responsables de la résorption osseuse, dépend de la présence de gènes exprimés dans les ostéoblastes, dont l'ostéoprotégérine et son ligand, et dans une certaine mesure l'interleukine 6 [3-7]. Cependant, un remodelage simultané dans plusieurs sites n'est pas incompatible avec une régulation endocrine. Contrôles local (autocrine et paracrine) et systémique (endocrine), loin d'être mutuellement exclusifs, peuvent au contraire être synergiques, comme on le voit pour plusieurs fonctions homéostatiques de l'organisme. Les preuves cliniques en faveur d'une régulation endocrine du remodelage osseux sont incontestables: les hormones stéroïdes sexuelles sont un maillon essentiel de la différenciation ostéoclastique et de la résorption osseuse [8]. Nous ne comprenons pas encore très bien leurs mécanismes d'action, mais il est clair que l'insuffisance des fonctions gonadiques entraîne une résorption osseuse pathologique chez l'humain, le rat et, à un moindre degré, chez la souris. D'autres hormones favorisent la résorption osseuse physiologique, telles l'hormone parathyroïdienne, dont le mécanisme d'action sur le squelette est incomplètement élucidé [9], et la calcitonine, qui possède des récepteurs sur les ostéoclastes [10]. L'essentiel des processus de régulation décrits ci-dessus ciblent les ostéoclastes, ce qui souligne un manque de connaissances surprenant en ce qui concerne le contrôle moléculaire de la formation osseuse par les ostéoblastes. A I'heure actuelle, la seule molécule connue pour influencer la vitesse de formation osseuse par les ostéoblastes, une fois ceux-ci différenciés, est le facteur de transcription Cbfal [11] (voir les articles de P. Ducy et de P. Marie, p. 1242 etp. 1252 de ce numéro). On serait cependant tenté de postuler l'existence d'un contrôle commun régissant à la fois la formation et la résorption osseuse. Une expérience in vivo suggère qu'il existe une régulation endocrine de la formation osseuse: des souris transgéniques pour le gène codant pour la thymidine kinase (tk) placé sous le contrôle d'un promoteur exprimé spécifiquement dans des ostéoblastes différenciés (souris osc-tk) [12] ont été traitées par ganciclovir. L'induction de l'expression du gène tk qui en résulte conduit à l'interruption de la réplication de I'ADN dans les cellules exprimant tk et à la mort cellulaire. Ce processus est réversible lors de l'arrêt du ganciclovir. Chez ces souris osc-tk traitées par ganciclovir, on ne détecte aucune formation osseuse, mais la résorption osseuse se poursuit de façon ininterrompue, ce qui aboutit à des os vides. II y a également un arrêt de croissance puisqu'une activité ostéoblastique est requise pour la croissance longitudinale du squelette. Mais, de façon tout à fait surprenante, lors de l'arrêt du traitement par le ganciclovir, la formation osseuse reprend, l'aspect des os se normalise, de même que la taille des souris et ce en un temps très court. L'aspect le plus remarquable de ce «rattrapage» de croissance est son extrême précision. Le volume osseux des souris osctk transgéniques est en effet identique à celui de leurs congénères sauvages. Cette précision extrême du processus de rattrapage, de même que sa vitesse, suggèrent que les ostéoblastes différenciés «savaient » combien de matrice osseuse devait être synthétisée dans différentes circonstances, et pouvaient le faire à petite ou à grande vitesse. Dans une première phase immédiatement après l'arrêt du ganciclovir, les ostéoblastes produisaient de grandes quantités de matrice osseuse, afin de combler le vide des os; puis, une fois atteint un volume osseux normal, la production de matrice osseuse se ralentissait, assurant simplement le maintien de ce volume osseux normal. Cette capacité des ostéoblastes d'évaluer précisément les besoins en synthèse protéique et d'adapter leur activité à ces besoins suggère l'existence d'un contrôle endocrinien de la formation osseuse.

\section{Contrôle commun de la masse osseuse, du poids corporel et de la reproduction: le rôle essentiel de la leptine}

Pour identifier des hormones susceptibles d'influencer la formation osseuse, nous avons utilisé les données de la littérature clinique. La perturbation la plus fréquente du remodelage osseux est l'ostéoporose, caractérisée par une augmentation relative de la résorption osseuse par rapport à la formation osseuse. Parmi les nombreux signes cliniques associés à cette maladie, deux nous ont paru intéressants car, ensemble, ils mettaient sur la piste d'un contrôle moléculaire de la formation osseuse accessible à l'expérimentation. II s'agit d'une part du lien qui existe entre insuffisance gonadique et perte osseuse et, d'autre part, entre obésité et protection contre cette même perte osseuse, même après la ménopause [13-17]. Ces observations suggèrent que trois paramètres, conservation de la masse osseuse, poids corporel et reproduction sont sous le contrôle d'une ou de plusieurs hormones communes.

\section{La leptine: \\ un régulateur du remodelage osseux}

La leptine, qui contrôle le poids corporel et la fonction de reproduction $[18,19]$, était certainement un sérieux candidat. L'existence de modèles animaux dont le système leptinique est déficient $(\mathrm{m} / \mathrm{s} 1998$, $n^{\circ} 8-9$, p. 845) a permis de tester in vivo, I'hypothèse d'un rôle de cette hormone dans le remodelage osseux. L'existence d'un hypogonadisme chez les souris ob/ob, déficientes en leptine et chez les souris $\mathrm{db} / \mathrm{db}$, sans récepteur fonctionnel, laissait prévoir une ostéopénie [20-26]. En effet, aucun modèle animal n'a jamais été décrit dans lequel co-existaient un hypogonadisme et une masse osseuse élevée. Aussi la découverte d'une masse osseuse élevée chez ces souris a été une surprise [27]. Les modifications phénotypiques étaient tellement importantes qu'une méthode 


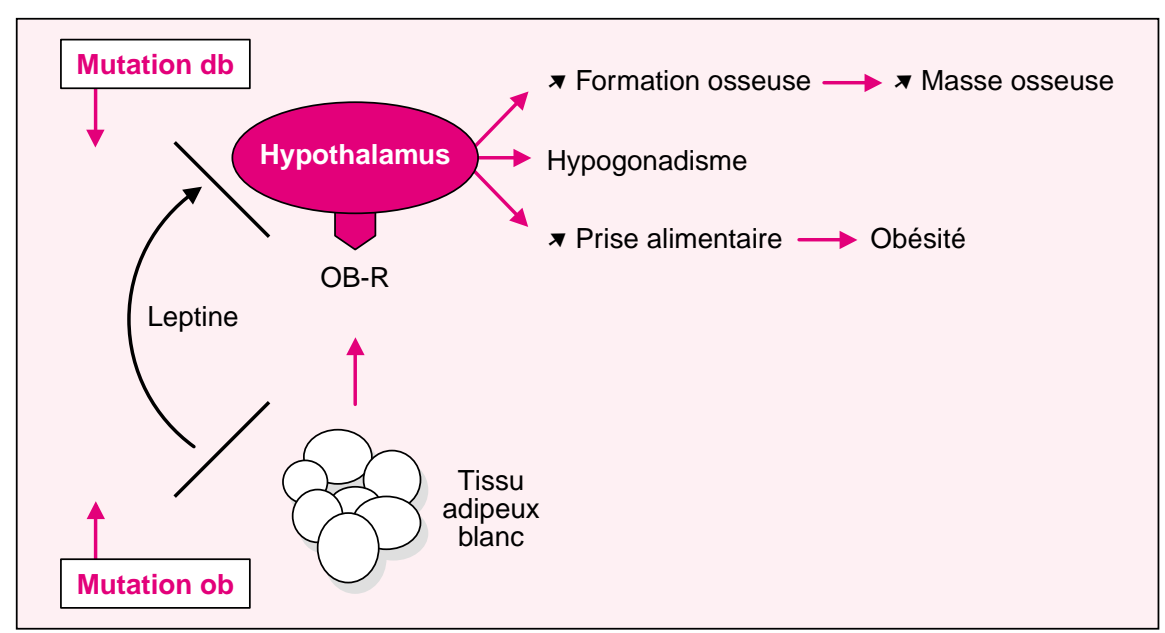

Figure 1. Conséquences des mutations ob/ob et $\mathbf{d b} / \mathbf{d b}$. La leptine est sécrétée par le tissu adipeux et agit sur l'hypothalamus par l'intermédiaire de son récepteur OB-R. La mutation ob résulte en l'absence de sécrétion de leptine fonctionnelle et la mutation $\mathrm{db}$, qui affecte $\mathrm{OB}-\mathrm{R}$, interrompt le signal leptinique. Ces deux types de mutation entraînent une augmentation de la prise alimentaire, un hypogonadisme et une augmentation de la masse osseuse, par des relais hypothalamiques qui ne sont pas encore entièrement décryptés.

de faible sensibilité, comme une radiographie, suffisait à les détecter. Par la suite, elles ont été confirmées par l'analyse histologique et histomorphométrique. Ces anomalies touchaient l'ensemble du squelette y compris les os longs, les vertèbres et le crâne, ce qui était parfaitement compatible avec un effet endocrinien. Les souris analysées n'étaient âgées que de six mois, mais avaient déjà atteint un stade d'obésité majeure. Les troubles de la masse osseuse auraient pû n'étre qu'une conséquence de l'obésité. Pour répondre à cette question, nous avons utilisé des souris ob/ob, soumises à un régime pauvre en graisses dès la naissance. On peut ainsi retarder l'apparition de l'obésité chez ces souris jusqu'à la sixième semaine de vie. Malgré ce régime et un poids corporel normal, ces souris ob/ob avaient déjà un phénotype de masse osseuse élevée, bien que moins prononcé que celui observé chez les animaux ob/ob adultes. Or, une telle augmentation de la masse osseuse n'était observée ni chez les souris jaunes Agouti, qui développent un phénotype d'obésité secondaire à la liaison de la protéine Agouti au récepteur 4 de la mélanocortine (MC-R4) [28], dont elle antagonise la voie de transduction [29] $(\mathrm{m} / \mathrm{s}$ p. 266), ni chez des souris sauvages soumises à un régime riche en graisses et en glucides pendant un mois pour induire un état d'obésité. Ces observations suggèrent donc I'implication directe du système leptinique dans l'augmentation de la masse osseuse.

Trois mécanismes possibles peuvent entraîner un phénotype de masse osseuse élevée. Une accélération de la différenciation et/ ou de la fonction ostéoblastique, une insuffisance de la fonction ostéoclastique, ou une combinaison des deux.

Nous avons abordé l'étude de la résorption osseuse chez les animaux déficients en leptine, de façon indirecte, par le biais de leur hypogonadisme. En effet, I'hypogonadisme s'accompagne toujours d'une augmentation du nombre des ostéoclastes, et cette règle générale vaut également pour les souris ob/ob et $\mathrm{db} / \mathrm{db}$. En rétablissant expérimentalement la fonction gonadique et un nombre normal d'ostéoclastes, on pouvait s'attendre à deux types de résultats: soit une aggravation $d u$ phénotype osseux, soit un phénotype inchangé en cas de déficience fonctionnelle des ostéoclastes en I'absence de leptine. Pour déterminer lequel de ces deux scénarios était correct, nous avons traité des souris ob/ob mâles et femelles avec des implants de testostérone ou d'estradiol pendant quatre mois. Ce traitement a corrigé I'hypogonadisme et diminué le nombre d'ostéoclastes, et a conduit à une aggravation considérable de l'excès de masse osseuse, éliminant ainsi I'hypothèse d'une anomalie de fonction des ostéoclastes secondaire à l'absence de leptine. Cette observation unique de la coexistence d'une masse osseuse élevée et d'un hypogonadisme nous a incité à rechercher le mécanisme général de l'action de la leptine dans le contrôle de la formation osseuse. Pour étudier la formation osseuse de façon dynamique, on peut se servir de I'histomorphométrie, une technique basée sur la fixation de la calcéine au front de minéralisation. Ainsi, ce composé, lorsqu'il est injecté à huit jours d'intervalle, permet de mesurer le débit d'apposition minérale, la vitesse de formation osseuse entre autres paramètres [30]. A I'aide de cette technique, nous avons pu démontrer une augmentation nette de tous les paramètres de formation osseuse chez les souris $o b / o b$ et $d b / d b$, bien avant la survenue de l'obésité. Tous les paramètres associés à la formation osseuse étaient augmentés, à l'exception cependant du nombre d'ostéoblastes qui était normal chez les souris $o b / o b$ et $d b / d b$, indiquant que la leptine n'affectait pas la prolifération des ostéoblastes, mais seulement leur fonction. Cette absence d'un effet mitogénique serait certainement un grand avantage si on envisage d'utiliser un antagoniste de la voie métabolique de la leptine pour favoriser la formation osseuse.

Nous avons vu que la masse osseuse élevée précède l'installation de l'obésité: elle pouvait donc, chez les souris $o b / o b$ et $d b / d b$, être secondaire à d'autres anomalies endocriniennes. Cette probabilité est faible car aucune hormone connue n'est capable de stimuler à ce point la formation osseuse chez les souris ob/ob eugonadiques. De fait, la plupart des anomalies endocriniennes observées chez les souris $o b / o b$ et $d b / d b$ n'ont qu'un faible retentissement sur la formation ou la résorption osseuse, à I'exception d'une seule: I'hypercorticisme. Mais une sécrétion accrue de glucocorticoïdes favorise la diminution, et non pas l'augmentation, de la formation osseuse [31, 32]. 


\section{Absence d'argument en faveur d'un effet direct, paracrine ou endocrine, de la leptine sur l'ostéoblaste in vivo}

Si l'augmentation de la masse osseuse observée en l'absence de leptine ou de sa voie de transduction cellulaire n'est pas la conséquence d'une anomalie endocrinienne secondaire, quel en est le mécanisme? La leptine a trois possibilités pour transmettre son signal à l'ostéoblaste, qui ne sont pas mutuellement exclusives: (1) une voie locale, impliquant un mécanisme autocrine et/ ou paracrine; (2) une voie endocrine classique; (3) enfin une voie neuro(endo)crine, par analogie avec le contrôle de l'appétit.

Puisque l'absence de leptine ne modifie pas le nombre d'ostéoblastes, elle doit en modifier la fonction. C'est pourquoi toutes les expériences visant à démontrer un effet autocrine, paracrine ou endocrine de la leptine ont utilisé des ostéoblastes différenciés, c'est-à-dire capables de synthétiser et de déposer de la matrice osseuse. L'hypothèse d'une secrétion autocrine de leptine a rapidement été éliminée au vu de I'absence de transcrits en N orthern blot dans l'os, ou dans des ostéoblastes en cultures primaires. L'intervention locale de la leptine par un mécanisme paracrine ou endocrine est, elle aussi, très peu probable, car nous n'avons pas été en mesure de détecter une expression quelconque du récepteur de la leptine $O b R b$ dans des cultures d'ostéoblastes primaires. II existe de multiples isoformes du récepteur de la leptine, mais seule l'isoforme appelée ObRb, exprimée principalement dans quatre noyaux hypothalamiques, a la capacité d'induire la voie de signalisation à travers la membrane cellulaire [20, 22]. L'absence d'O bRb dans les ostéoblastes primaires était en contradiction avec les résultats de Thomas et al., obtenus en utilisant des cellules transformées, et fondés sur une analyse par RT-PCR toujours sujette à caution [33]. Cette divergence soulignait l'importance d'une approche biochimique, génétique et physiologique.

Un autre argument expérimental infirmait I'hypothèse d'une action directe de la leptine sur les ostéo- blastes: diverses concentrations de leptine, allant de 8 à $100 \mathrm{ng} / \mathrm{ml}$ étaient incapables d'induire dans des ostéoblastes primaires les intermédiaires de la voie de signalisation activée par $\mathrm{ObRb}$ que sont la phosphorylation de STAT3 et I'augmentation de l'expression des gènes de réponse précoce, comme Tisl1 et C-fos [3438]. Au contraire, I'oncostatine M, induisait invariablement une phosphorylation de STAT 3 et une expression de Tisll et de $C$-fos. Or le récepteur de l'oncostatine $M$ appartient à la même superfamille que le récepteur de la leptine, est présent en abondance à la surface d'ostéoblastes différenciés [39], et son activation conduit également à la phosphorylation de STAT 3 et à l'expression de gènes de réponse précoce $[40,41]$. Les données génétiques confirmaient également que l'ostéoblaste n'est pas une cible directe de la leptine. Si le phénotype osseux des souris ob/ob et des souris $\mathrm{db} / \mathrm{db}$ était principalement dû à l'absence de récepteurs de la leptine impliqués dans la transduction du signal, au niveau de la surface d'ostéoblastes différenciés, des cultures d'ostéoblastes primaires de souris $\mathrm{db} / \mathrm{db}$, ayant une mutation inactivatrice d'ObRb, devraient produire plus de matrice osseuse que les cultures d'ostéoblastes primaires provenant de souris sauvages. Cela n'est pas le cas [42]. L'ensemble de ces arguments, certes indirects, indiquent néanmoins qu'un effet local de la leptine ne peut rendre compte de son action

\section{Contrôle de la formation osseuse in vivo par la leptine: un mécanisme central}

La recherche infructueuse d'un effet endocrine ou paracrine de la leptine sur l'os, combinée à ce que l'on connaît de l'implication de cette hormone dans le contrôle du poids corporel et du déclenchement de la puberté nous ont amenés à envisager que contrôle de la masse osseuse par cette hormone procède d'un mécanisme neuro-endocrinien. Prouver cette hypothèse nécessitait une double approche génétique et physiologique: (1) la perfusion intracérébroventriculaire de leptine à une concentration telle que I'hormone ne traverse pas la barrière cérébro- méningée, devait corriger le phénotype de masse osseuse élevée chez les souris ob/ ob; (2) la même perfusion intra-cérébroventriculaire de leptine, mais cette fois chez des souris sauvages, devait conduire à une ostéopénie.

L'administration à des souris ob/ob par voie intra-cérébroventriculaire de doses faibles de leptine ( $8 \mathrm{ng} / \mathrm{h}$ ) induisent chez ces animaux une perte de poids corporel considérable, comme cela a déjà été montré antérieurement. La dose de leptine que nous avons administrée était la même que celle utilisée pour démontrer que la leptine agissait par une voie centrale pour contrôler le poids corporel [43] ; or nous nous sommes assurés qu'il n'y avait pas de leptine détectable dans le sérum de ces animaux. Le traitement par perfusion intra-cérébroventriculaire a été prolongé un mois, un temps relativement long en termes de contrôle du poids corporel mais relativement court en terme de remodelage osseux, puisqu'un cycle de renouvellement osseux peut durer plus de trois mois. Néanmoins, à la fin de cette période d'un mois, les animaux ob/ ob traités par de la leptine avaient une masse osseuse légèrement inférieure à celle des animaux sauvages. On peut donc conclure que la leptine peut modifier la formation osseuse alors même qu'elle n'est présente ni dans le sérum, ni dans le micro-environnement osseux, ce que confirme également l'absence d'induction de la voie de signalisation activée par $\mathrm{ObRb}$ dans les ostéoblastes issus de cultures primaires. On est donc en présence d'un nouveau mode de régulation du remodelage osseux, qui met en jeu un contrôle central. Ce contrôle central intervient aussi chez des animaux normaux. De fait, la perfusion intracérébroventriculaire de la même dose faible de leptine chez les souris sauvages entraîne une ostéopénie, dans un laps de temps d'un mois, ce qui démontre la réalité physiologique de la modulation du remodelage osseux par la leptine [27].

Mais comment agit la leptine en aval ? II n'existe pas encore de modèle clair et simple pour expliquer comment la leptine contrôle l'appétit, à la suite de sa fixation sur son ObRb dans I'hypothalamus [44, 
45]. II faut admettre que notre compréhension du mode d'action de la leptine dans la formation osseuse n'est pas plus avancée. U ne première approche consiste à déterminer si les neuropeptides qui modulent l'action de la leptine sur le contrôle du couple appétit/ poids corporel agissent aussi sur le contrôle par la leptine de la masse osseuse. Par exemple, le neuropeptide Y (NPY) est l'un des nombreux neuropeptides qui stimulent l'appétit et la prise de poids corporel. L'expression de NPY est augmentée chez les souris ob/ob et un déficit en NPY corrige partiellement le phénotype d'obésité de ces souris ob/ ob [46, 47]. La leptine et le NPY ont donc des effets contraires en ce qui concerne le contrôle du poids corporel. Tel n'est pas le cas pour le contrôle de la formation osseuse: la perfusion intra-cérébroventriculaire de NPY chez des souris sauvages induisait une perte osseuse, comme le fait la leptine. II est donc probable que la leptine utilise différents médiateurs pour contrôler le poids corporel et la masse osseuse.

\section{Implications}

L'observation que la leptine module la formation de l'os par un mécanisme neuroendocrinien a d'importantes implications pour la biologie du squelette. Les observations faites chez la souris n'établissent pas seulement un nouveau concept physiologique et la base moléculaire de l'effet protecteur bien connu de l'obésité pour la masse osseuse, elles ouvrent également de nouvelles voies de recherche en physiologie et pharmacologie. On peut se demander pourquoi des vertébrés ont besoin d'un système aussi puissant pour garantir la formation osseuse? La réponse à cette question est naturellement très spéculative. Bien que les conséquences d'un contrôle imparfait du remodelage osseux ne s'expriment pas aussi rapidement que celles d'un contrôle imparfait du poids corporel, on peut émettre I'hypothèse qu'il s'agit là d'une fonction critique dont la régulation doit être étroitement assurée durant l'évolution. Nous savons déjà que des anomalies du contrôle de la résorption osseuse peuvent entraîner une ostéopétrose, mière enfance. De même, des modèles expérimentaux entraînant un accroissement non contrôlé de la formation osseuse ont pour conséquences l'incapacité de la moelle osseuse à maintenir une hématopoï̀se normale et des anomalies du bilan phosphocalcique comprenant une baisse de la calcémie, l'ensemble de ces anomalies entraînant une mort précoce [48]. Si la régulation de la formation osseuse est importante à ce point il est alors probable que la leptine ne soit pas le seul acteur et qu'il existe également une modulation positive de la formation osseuse dont le rôle serait de contrer I'action de la leptine. De même, si on se range à une telle vue globale du remodelage osseux on peut concevoir et tester I'hypothèse selon laquelle la résorption, autre versant du remodelage osseux, serait également soumise à un contrôle hormonal central.

Finalement, s'il est encore trop tôt pour savoir si ce nouveau concept de régulation du remodelage osseux conduira à des approches thérapeutiques nouvelles, cette voie mérite certainement d'être explorée. Comme cela a été discuté dans cette revue, deux aspects de la modulation de la masse osseuse par la leptine retiennent I'attention dans cette perspective. Le premier est que la modulation de la voie de signalisation de la leptine offre la possibilité d'augmenter la formation de la masse osseuse, qui est véritablement déficitaire dans l'ostéoporose. On en conçoit aisément l'intérêt dans un traitement curatif, ou même un traitement préventif, de l'ostéoporose. Le second aspect est que la stimulation de la formation osseuse en l'absence de leptine ou de sa voie de signalisation intervient sans que le nombre des ostéoblastes augmente, ce qui est un avantage potentiel majeur si l'on envisage un traitement médical au long cours

\section{RÉFÉRENCES}

1. Karsenty G. The genetic transformation of bone biology. Genes Dev 1999; 13: 303751

2. Manolagas SC. Birth and death of bone cells: basic regulatory mechanisms and implications for the pathogenesis and treat- ment of osteoporosis. Endocrinol Rev 2000; 2: 115-37.

3. Simonet WS, Lacey DL, Dunstan CR, et al. Osteoprotegerin : a novel secreted protein involved in the regulation of bone density. Cell 1997; 89: 309-19.

4. Bucay N, Sarosi I, Dunstan CR, et al. Osteoprotegerin-deficient mice develop early onset osteoporosis and arterial calcification. G enes Dev 1998; 12 : 1260-8.

5. Lacey DL, Timms E, Tan HL, et al. Osteoprotegerin ligand is a cytokine that reguates osteoclast differentiation and activation. Cell 1998; 93: 165-76.

6. Kong YY, Yoshida H, Sarosi I, et al. OPGL is a key regulator of osteoclastogenesis, lymphocyte development and lymph-node organogenesis. Nature 1999; 397: 315-23.

7. Poli V, Balena R, Fattori E, et al. Interleukin-6 deficient mice are protected from bone loss caused by estrogen depletion. EM BO J 1994; 5 : 1189-96.

8. Couse JF, Korach KS. Estrogen receptor null mice: what have we learned and where will they lead us? Endocrinol Rev 1999; 3 : 358-417.

9. Potts JT, and Juppner H. Parathyroid hormone and parathyroid hormone-related peptide in calcium homeostasis, bone metabolism and bone development: the proteins, their genes, and receptors In: Avioli LV, Krane SM, eds. M etabolic bone disease and clinically related disorders. San Diego; Academic Press, 1998.

10. Nicholson GC Moseley JM, Sexton PM, et al. Abundant calcitonin receptors in isolated rat osteoclasts. Biochemical and autoradiographic characterization. J Clin Invest 1986 ; 2 : 355-60.

11. Ducy P, Starbuck M, Priemel M, et al. A Cbfal-dependent genetic pathway controls bone formation beyond embryonic development. Genes Dev 1999; 13: 1025-36.

12. Corral DA, Amling $M$, Priemel $M$, et al. Dissociation between bone resorption and bone formation in osteopenic transgenic mice. Proc Natl Acad Sci USA 1998; 95 : 13835-40.

13. Felson DT, Zhang $Y, H$ annan $M T$, et al. Effects of weight and body mass index on bone mineral density in men and women: The Framingham study. I Bone $M$ iner Res $1993 ; 8$ : 567-73.

14. Ravn P, Cizza G, Bjarnason NH, et al. Low body mass index is an important risk factor for low bone mass and increased bone loss in early postmenopausal women early postmenopausal intervention cohort (EPIC) study group. J Bone M in Res 999; 14 : 1622-7.

15. Riggs BL, M elton L $3 \mathrm{~d}$ : involutional osteoporosis. N Engl J M ed 1986; 314: 1676-86.

16. Riggs BL, Khosla S, Melton LJ. $3^{\text {rd }}$. A unitary model for involutional osteoporosis: estrogen deficiency causes both type I and type 11 osteoporosis in postmenopausal women and contributes to bone loss in aging men. J Bone M iner Res 1998; 13: 76373. 


\section{RÉFÉRENCES}

17. Tremollieres FA, Pouilles JM, Ribot C. Vertebral post-menopausal bone loss is reduced in overweight women : a longitudinal study in 155 early postmenopausal women. Clin Endocrinol M etab 1993; 77 : 683-6.

18. Friedman JM, Halaas JL. Leptin and the regulation of body weight in mammals. Nature 1998; 395: 763-70.

19. Spiegelman BM, Flier JS. Adipogenesis and obesity: rounding out the big picture. Cell 1996; 87 : 377-89.

20. Chen $\mathrm{H}$, Charlat $\mathrm{O}$, Tartaglia $L A$, et al. Evidence that the diabetes gene encodes the leptin receptor: identification of a mutation in the leptin receptor gene in $\mathrm{db} / \mathrm{db}$ mice. Cell 1996; 84: 491-5.

21. Clement K, Vaisse C, Lahlou N, et al. A mutation in the human leptin receptor gene causes obesity and pituitary dysfunction. Nature 1998; 392: 398-401.

22. Lee $G H$, Proenca $R$, Montez JM, et al. Abnormal splicing of the leptin receptor in diabetic mice. Nature 1996; 379: 632-5.

23. Montague CT, Faroogi IS, Whitehead JP, et al. Congenital leptin deficien cy is associated with severe early-onset obesity in humans. Nature 1997; 387: 903-8.

24. Strobel A, Issad T, Camoin $L$, et al. A leptin missense mutation associated with hypogonadism and morbid obesity. Nat Genet 1998; 3: 213-5.

25. Tartaglia LA, Dembski $M$, Weng $X$, \& al. Identification and expression cloning of a leptin receptor, OB-R. Cell 1995; 83: 1263-71.

26. Zhang $Y$, Proenca $R$, Maffei $M$, et al. Positional cloning of the mouse obese gene and its human homologue Nature 1994 372 : 425-32 et $1995 ; 374: 479$ (erratum).

27. Ducy P, Amling M, Takeda S, et al. Leptin inhibits bone formation through a hypothalamic relay: a central control of bone mass. Cell 2000; 2: 197-207.

28. Dinulescu DM, Cone RD. Agouti and agouti-related protein : an alogies and contrasts. J Biol Chem 2000; 275: 6695-8.

29. Lu D, Willard D, Patel IR, et al. Agouti protein is an antagonist of the melanocytestimulating-hromone receptor. Nature 1994 371: 799-802.

30. Parfitt AM, Drezner MK, Glorieux FH, et al. Bone histomorphometry: standardization of nomenclature symbols, and units Report of the ASBMR histomorphometry nomenclature committee. J Bone $M$ iner Res $1987 ; 6$ : 595-610.

31. Ahima RS, Prabakaran D, Mantzoros C, et al. Role of leptin in the neuroendocrine response to fasting. Nature $1996 ; 382$ : 25052

32. Reid IR. Preventing glucocorticoid-induced osteoporosis. N Engl J M ed 1997; 337: 420-1.

$\mathrm{m} / \mathrm{s} \mathrm{n}^{\circ} 12$, vol. 17, décembre 2001
33. Thomas T, Gori F, Khosla S, et al. Leptin acts on human marrow stromal cells to enhance differentiation to osteoblasts and to inhibit differentiation to adipocytes. Endocrinology 1999; 140: 1630-8.

34. Baumann H, Morella KK, White DW, et al. The full-length leptin receptor has signaling capabilities of interleukin 6-type cytokine receptors. Proc Natl A cad Sci USA 1996; 93: 8374-5.

35. Elmquist JK, Ahima RS, Maratos-Flier E, et al. Leptin activates neurons in ventrobasal hypothalamus and brainstem. Endocrinology 1997: 138: 839-42.

36. Ghilardi N, Ziegler S, Wiestner A, et al. Defective STAT signaling by the leptin receptor in diabetic mice. Proc N atl Acad Sci USA 1996; 93: 6231-5.

37. Moitra J, Mason MM, Olive $M$, et al. Life without white fat: a transgenic mouse. Genes Dev 1998; 12 : 3168-81.

38. Vaisse $\mathrm{C}, \mathrm{H}$ alaas $\mathrm{L}, \mathrm{H}$ orvath $\mathrm{CM}$, et al. Leptin activation of Stat3 in the hypothalamus of wild-type and ob/ob mice but not $\mathrm{db} / \mathrm{db}$ mice. Nat Genet 1996; 14: 95-7.

39. Ahn S, Olive M, Aggarwal $S$, et al. A dominant-negative inhibitor of CREB reveals that it is a general mediator of stimulus-dependent transcription of C-fos. $\mathrm{M} \mathrm{Ol}$ Cell Biol 1998; 2: 967-77.

40. Lew JB, Schindler C, Raz R, et al. Activation of the JAK-STAT signal transduction pathway by oncostatin-M cultured human and mouse osteoblastic cells. Endocrinology 1996; 137: 1159-65.

41. Bellido T, Stahl N, Farruggella TJ, \& al. Detection of receptors for interleukin-6, interleukin-11, leukemia inhibitory factor, oncostatin $M$, and ciliary neurotrophic factor in bone marrow stromal/ osteoblastic cells. J Clin Invest 1996; 2: 431-7.

42. Westvik J. Radiological features in generalized lipodystrophy. Acta Paediatr 1996; 413 (suppl) : 44-51.

43. Halaas JL, Boozer C, Blair-West J, et al. Physiological response to long-term peripheral and central leptin infusion in lean and obese mice. Proc Natl Acad Sci USA 1997; 94 : 8878-83.

44. Elmquist JK, Elias CF, Saper CB. From lesions to leptin: hypothalamic control of food intake and body weight. Neuron 1999; 2: 221-32.

45. Marsh DJ, Hollopeter G, Huszar D, et al. Response of melanocortin-4 receptor-deficient mice to anorectic and orexigenic peptides. Nat Gen et $1999 ; 1: 119-22$.

46. Erickson JC, H ollopeter G, Palmiter RD. Attenuation of the obesity syndrome of $\mathrm{ob} / \mathrm{ob}$ mice by the loss of neuropeptide $\mathrm{Y}$. Science 1996; 274 : 1704-7.

47. Schwartz MW, Erickson JC, Baskin DG, et al. Effect of fasting and leptin deficiency on hypothalamic neuropeptide $Y$ gene transcription in vivo revealed by expression of a lacZ reporter gene. Endocrinology 1998; 5: 2629-35.
48. Jochum W, David JP, Elliott C, et al. Increased bone formation and osteosclerosis in mice overexpressing the transcriptin factor fra-1. N at M ed $2000 ; 6$ : 980-4.

\section{Summary}

Leptin controls bone formation through a hypothalamic relay

Menopause favors osteoporosis and obesity protects from it. In an attempt to decipher the molecular bases of these two well-known clinical observations, we hypothesized that they meant that bone remodeling, body weight, and reproduction are controlled by identical endocrine pathways. We used mouse genetics as a tool to translate these clinical observations into a molecular hypothesis. The $\mathrm{ob} / \mathrm{ob}$ and $\mathrm{db} / \mathrm{db}$ mice were valuable models, since two of the three functions thought to be coregulated are affected in these mice: they are obese and hypogonadic. Surprisingly, given their hypogonadism, both mouse mutant strains have a high bone mass phenotype. Subsequent anaIysis of the mechanism leading to this high bone mass revealed that this was due to an increase of bone formation. All data collected indicate that, in vivo, leptin does not act directly on osteoblasts but rather through a central pathway following binding to its specific receptors located on hypothalamic nuclei. This result revealed that bone remodeling, like most other homeostatic functions, is under a hypothalamic control. The nature of the signal downstream of the hypothalamus is unknown for now but current experiments are attempting to identify it.

\section{TIRÉS À PART}

\section{G. Karsenty.}

